\title{
ON A FUNCTIONAL VOLTERRA-FREDHOLM INTEGRAL EQUATION, VIA PICARD OPERATORS
}

\author{
Nicolaie LUngu AND IOAN A. Rus
}

Abstract. In this paper we present some results relative to existence, uniqueness, integral inequalities and data dependence for the solutions of the functional Volterra-Fredholm integral equation with deviating argument in a Banach space:

$$
u(x, y)=g(x, y, h(u)(x, y))+\int_{0}^{x} \int_{0}^{y} K(x, y, s, t, u(s, t)) d s d t, \quad x, y \in \mathbb{R}_{+}
$$

by Picard operators technique. This equation is a generalization of the equation (VF) from the paper: B.G. Pachpatte, On Volterra-Fredholm integral equation in two variables, Demonstratio Math., 40(2007), No. 4, 832-852.

\section{Introduction}

The present paper is motivated by a recent paper [5] by B.G. Pachpatte which studies a system of Volterra-Fredholm integral equations in two variables $x, y \in \mathbb{R}_{+}$. The aim of our paper is to study the following more general Volterra-Fredholm integral equation with deviating argument in a Banach space:

$$
u(x, y)=g(x, y, h(u)(x, y))+\int_{0}^{x} \int_{0}^{y} K(x, y, s, t, u(s, t)) d s d t, \quad x, y \in \mathbb{R}_{+} .
$$

Let $(E,|\cdot|)$ be a Banach space. Let $\tau>0$ and

$$
X_{\tau}:=\left\{u \in C\left(\mathbb{R}_{+}^{2}, E\right)|\exists M(u)>0:| u(x, y) \mid e^{-\tau(x+y)} \leqslant M(u)\right\} .
$$

On $X_{\tau}$ we consider Bielecki's norm

$$
\|u\|_{\tau}:=\sup _{x, y \in \mathbb{R}_{+}}\left(|u(x, y)| e^{-\tau(x+y)}\right) .
$$

It is clear that $\left(X_{\tau},\|\cdot\|_{\tau}\right)$ is a Banach space.

Mathematics subject classification (2000): 45G10, 47H10, 45N05, 47N20.

Keywords and phrases: Volterra-Fredholm functional integral equations, contraction principle, Picard operator, existence and uniqueness, data dependence. 
Relative to (1.1) we suppose that:

(c $\left.c_{1}\right) \quad g \in C\left(\mathbb{R}_{+}^{2} \times E, E\right), K \in C\left(\mathbb{R}_{+}^{4} \times E, E\right) ;$

$\left(c_{2}\right) \quad h: X_{\tau} \rightarrow X_{\tau}$ is such that

$$
\begin{gathered}
\exists l_{h}>0: \quad|h(u)(x, y)-h(v)(x, y)| \leqslant l_{h}\|u-v\|_{\tau} \cdot e^{\tau(x+y)}, \\
\forall x, y \in \mathbb{R}_{+}, \forall u, v \in X_{\tau} ;
\end{gathered}
$$

(c) $\exists l_{g}>0: \quad\left|g\left(x, y, e_{1}\right)-g\left(x, y, e_{2}\right)\right| \leqslant l_{g}\left|e_{1}-e_{2}\right|$,

$$
\forall x, y \in \mathbb{R}_{+}, \forall e_{1}, e_{2} \in E ;
$$

$\left(c_{4}\right) \quad \exists l_{K}(x, y, s, t): \quad\left|K\left(x, y, s, t, e_{1}\right)-K\left(x, y, s, t, e_{2}\right)\right|$

$$
\leqslant l_{K}(x, y, s, t)\left|e_{1}-e_{2}\right|, \forall x, y, s, t \in \mathbb{R}_{+}, e_{1}, e_{2} \in E ;
$$

(c) $l_{K} \in C\left(\mathbb{R}_{+}^{4}, \mathbb{R}_{+}\right)$and

$$
\int_{0}^{x} \int_{0}^{y} l_{K}(x, y, s, t) e^{\tau(s+t)} d s d t \leqslant l e^{\tau(x+y)}, \forall x, y \in \mathbb{R}_{+} ;
$$

(c) $\quad l_{h} l_{g}+l<1$.

We consider the operator

$$
A: X_{\tau} \rightarrow X_{\tau}, \quad A(u)(x, y):=\text { second part of (1.1). }
$$

First of all we shall prove that under the conditions $\left(c_{1}\right)-\left(c_{6}\right)$ the operator $A$ is a Picard operator. So, we present some notions and results from Picard operators theory (see I.A. Rus [6]-[8]).

\section{Picard operators}

Let $(X, d)$ be a metric space, $A: X \rightarrow X$ an operator and $c>0$. We denote by $F_{A}$ the fixed point set of $A$.

Definition 2.1. (I. A. Rus [6]-[8]) $A$ is a Picard operator (PO) if there exists $x_{A}^{*} \in X$ such that:

(i) $F_{A}=\left\{x_{A}^{*}\right\}$;

(ii) $A^{n}(x) \rightarrow x_{A}^{*}$ as $n \rightarrow \infty, \forall x \in X$.

Definition 2.2. (I. A. Rus [8]) $A$ is a c-Picard operator (c-PO) if $A$ is PO and $d\left(x, x_{A}^{*}\right) \leqslant c d(x, A(x)), \forall x \in X$.

LEMMA 2.1. (Abstract Gronwall lemma) ([8]) Let $(X, d, \leqslant)$ be an ordered metric space and $A: X \rightarrow X$ an operator. We suppose that

(i) $A$ is $P O$;

(ii) A is increasing.

If we denote by $x_{A}^{*}$ the unique fixed point of $A$, then

(a) $x \leqslant A(x) \Rightarrow x \leqslant x_{A}^{*}$;

(b) $x \geqslant A(x) \Rightarrow x \geqslant x_{A}^{*}$. 
LEMMA 2.2. (I. A. Rus [8]) Let $(X, d, \leqslant)$ be an ordered metric space and $A, B, C$ : $X \rightarrow X$ be such that

(i) $A \leqslant B \leqslant C$;

(ii) The operators $A, B, C$ are POs;

(iii) The operator $B$ is increasing. Then

$$
x_{A}^{*} \leqslant x_{B}^{*} \leqslant x_{C}^{*}
$$

\section{Existence and uniqueness}

We begin with

THEOREM 3.1. Under the conditions $\left(c_{1}\right)-\left(c_{6}\right)$ the equation (1.1) has in $X_{\tau}$ a unique solution $u^{*}$ and the sequence of successive approximations

$$
u_{n+1}(x, y)=g\left(x, y, h\left(u_{n}\right)(x, y)\right)+\int_{0}^{x} \int_{0}^{y} K\left(x, y, s, t, u_{n}(s, t)\right) d s d t, \quad n \in \mathbb{N}
$$

converges uniformly to $u^{*}$.

Proof. We have that the operator $A$ is a contraction in $X_{\tau}$ with respect to $\|\cdot\|_{\tau}$. Indeed, for $u, v \in X_{\tau}$ from $\left(c_{1}\right)-\left(c_{6}\right)$ it follows

$$
\begin{aligned}
|A(u)(x, y)-A(v)(x, y)| \leqslant & |g(x, y, h(u)(x, y))-g(x, y, h(v)(x, y))| \\
& +l_{g}|h(u)(x, y)-h(v)(x, y)| \\
& +\int_{0}^{x} \int_{0}^{y} l_{K}(x, y, s, t)|u(s, t)-v(s, t)| d s d t \\
\leqslant & l_{g} l_{h}\|u-v\|_{\tau} e^{\tau(x+y)}+l\|u-v\|_{\tau} e^{\tau(x+y)} \\
\leqslant & \left(l_{g} l_{h}+l\right) e^{\tau(x+y)}\|u-v\|_{\tau} .
\end{aligned}
$$

Hence

$$
\|A(u)-A(v)\|_{\tau} \leqslant\left(l_{g} l_{h}+l\right)\|u-v\|_{\tau}
$$

for all $u, v \in X_{\tau}$.

From $\left(c_{6}\right)$ we have that $A$ is a contraction. So, from the contraction principle $A$ is a c-Picard operator, where

$$
c=\frac{1}{1-l_{g} l_{h}-l}
$$

REMARK 3.1. If $E:=\mathbb{R}^{n}$ and

$$
g(x, y, h(u)(x, y)):=h(x, y)+\int_{0}^{\infty} \int_{0}^{\infty} G(x, y, s, t, u(s, t)) d s d t
$$

then we have Pachpatte's result. 
REMARK 3.2. If $E:=l^{p}(\mathbb{R}), 1 \leqslant p<\infty$, then the equation (1.1) is an infinite system of functional-integral equations: $u_{i}(x, y)=g_{i}\left(x, y, h\left(u_{1}, \ldots, u_{n}, \ldots\right)(x, y)+\int_{0}^{x} \int_{0}^{y} K_{i}\left(x, y, s, t, u_{1}(s, t), \ldots, u_{n}(s, t), \ldots\right) d s d t\right.$ for all $x, y \in \mathbb{R}_{+}$and $i \in \mathbb{N}^{*}$.

\section{Integral inequalities}

THEOREM 4.1. Let $(E,|\cdot|, \leqslant)$ be an ordered Banach space. We suppose that:

(i) the conditions $\left(c_{1}\right)-\left(c_{6}\right)$ are satisfied;

(ii) the operators

$$
\begin{aligned}
& g(x, y, \cdot): E \rightarrow E \\
& h: E \rightarrow E \\
& K(x, y, s, t, \cdot): E \rightarrow E
\end{aligned}
$$

are increasing.

If $u^{*} \in X_{\tau}$ is the unique solution of the equation (1.1) and $u \in X_{\tau}$ is a solution of the following inequality

$$
u(x, y) \leqslant g(x, y, h(u)(x, y))+\int_{0}^{x} \int_{0}^{y} K(x, y, s, t, u(s, t)) d s d t, \forall x, y \in \mathbb{R}_{+}
$$

then

$$
u(x, y) \leqslant u^{*}(x, y)
$$

Proof. We consider the operator

$$
A: X_{\tau} \rightarrow X_{\tau}, \quad A(u)(x, y):=\text { second part of (1.1). }
$$

The operator $A$ is a Picard operator. From the condition (ii) $A$ is increasing. Then the proof follows from Lemma 2.1 .

EXAMPle 4.1. Consider:

$$
g(x, y, h(u)(x, y)) \leqslant h(x, y)
$$

and

$$
K(x, y, s, t, u(s, t)) \leqslant b(x, y) L(s, t, u(s, t)),
$$

where $h, b: \mathbb{R}_{+}^{2} \rightarrow \mathbb{R}_{+}$are continuous functions and $L: \mathbb{R}_{+}^{3} \rightarrow \mathbb{R}_{+}$is a continuous function which satisfies the condition

$$
0 \leqslant L(x, y, v)-L(x, y, w) \leqslant M(x, y, w)(v-w)
$$

for $x, y \in \mathbb{R}_{+}$and $v \geqslant w \geqslant 0 . M$ is a nonnegative continuous function defined on $\mathbb{R}_{+}^{3}$. 
Then

$$
u(x, y) \leqslant h(x, y)+b(x, y) \int_{0}^{x} \int_{0}^{y} L(s, t, u(s, t)) d s d t .
$$

Consider the operator $B: X_{\tau} \rightarrow X_{\tau}, B(u)(x, y):=$ last part of (4.3).

It is clear that the operator $B$ is PO on $X_{\tau}$ and is increasing.

Let $u_{B}^{*}$ be the unique fixed point of $B$. Thus, from Lemma 2.2, we have

$$
u^{*}(x, y)=A\left(u^{*}\right) \leqslant B\left(u_{B}^{*}\right)=u_{B}^{*} .
$$

From the papers [1]+[2] (S.S. Dragomir and N.M. Ionescu), we have

$$
u_{B}^{*}(x, y)=h(x, y)+b(x, y)\left[\exp \left(\int_{0}^{x} \int_{0}^{y} P(s, t) d s d t\right)-1\right]
$$

where

$$
P(x, y)=\left[L^{2}(x, y, h(x, y))+M^{2}(x, y, h(x, y)) b^{2}(x, y)\right]^{1 / 2},
$$

for $x, y \in \mathbb{R}_{+}$.

Then

$$
u(x, y) \leqslant u_{B}^{*}(x, y)
$$

\section{Data dependence: Monotony}

Consider the following integral equations

$u_{i}(x, y)=g_{i}(x, y, h(u)(x, y))+\int_{0}^{x} \int_{0}^{y} K_{i}(x, y, s, t, u(s, t)) d s d t, \quad i=1,2,3$.

THEOREM 5.1. We suppose that

(i) $g_{i}, h, K_{i}, i=1,2,3$ satisfy the conditions $\left(c_{1}\right)-\left(c_{6}\right)$;

(ii) $g_{1} \leqslant g_{2} \leqslant g_{3}, K_{1} \leqslant K_{2} \leqslant K_{3}$;

(iii) the operators:

$$
\begin{aligned}
& g_{2}(x, y, \cdot): E \rightarrow E \\
& h: E \rightarrow E \\
& K_{i}(x, y, s, t, \cdot): E \rightarrow E, i=1,2,3
\end{aligned}
$$

are increasing.

Then the equation (5.1i) has a unique solution $u_{i}^{*}$ and

$$
u_{1}^{*} \leqslant u_{2}^{*} \leqslant u_{3}^{*}
$$

Proof. Consider the operators $A_{i}, i=1,2,3, A_{i}: X_{\tau} \rightarrow X_{\tau}$

$$
A_{i}(u)(x, y):=\text { second part of (5.1i), } \quad i=1,2,3 \text {. }
$$

These operators are as in Lemma 2.2. We remark that $u_{i}^{*} \in X_{\tau}, i=1,2,3$. The proof follows from Lemma 2.2. 


\section{Data dependence: Continuity}

In this section we shall use the following notations:

$$
\widetilde{g}(u)(x, y):=g(x, y, h(u)(x, y))
$$

and

$$
\widetilde{K}(u)(x, y):=\int_{0}^{x} \int_{0}^{y} K(x, y, s, t, u(s, t)) d s d t .
$$

Let us consider the following equations

$u_{i}(x, y)=g_{i}(x, y, h(u)(x, y))+\int_{0}^{x} \int_{0}^{y} K_{i}(x, y, s, t, u(s, t)) d s d t, \quad i=1,2$.

We have

THEOREM 6.1. We suppose that:

(i) $g_{i}, h, K_{i}, i=1,2$, satisfy the conditions $\left(c_{1}\right)-\left(c_{6}\right)$;

(ii) there exist $\eta_{i} \in \mathbb{R}_{+}, i=1,2$, such that

$$
\left\|\widetilde{g}_{1}(u)-\widetilde{g}_{2}(u)\right\|_{\tau} \leqslant \eta_{1} \text { and }\left\|\widetilde{K}_{1}(u)-\widetilde{K}_{2}(u)\right\|_{\tau} \leqslant \eta_{2}, \forall u \in X_{\tau} .
$$

Then, if $u_{i}^{*}$ is the unique solution of (6.1i), then:

$$
\left\|u_{1}^{*}-u_{2}^{*}\right\| \leqslant \eta_{1}+\eta_{2} .
$$

Proof. The proof follows from Theorem 7.1.1 in I.A. Rus [7].

\section{Data dependence: Differentiability}

In this section we take $E=\mathbb{R}$.

To study the differentiability of the fixed point with respect to parameters we need the fiber contraction theorem.

THEOREM 7.1. (Fiber contraction theorem [3], [4], [7]) Let $(X, d)$ be a metric space and $(Y, \rho)$ be a complete metric space.

Let $B: X \times Y \rightarrow X \times Y$ be a continuous operator and $A: X \rightarrow X, \mathscr{D}: X \times Y \rightarrow Y$ two operators. We suppose that:

(i) $B(u, w)=(A(u), \mathscr{D}(u, w))$ for all $u \in X, w \in Y$,

(ii) $A$ is a Picard operator;

(iii) there exists $q \in(0,1)$ such that

$$
\rho(\mathscr{D}(u, w), \mathscr{D}(u, \bar{w})) \leqslant q \rho(w, \bar{w})
$$

for all $u \in X$ and $w, \bar{w} \in Y$.

Then $B$ is a Picard operator.

In what follows we consider the equation $u(x, y, \lambda)=g(x, y, h(u)(x, y), \lambda)+\int_{0}^{x} \int_{0}^{y} K(x, y, s, t, u(s, t, \lambda), \lambda) d s d t, \forall x, y \in \mathbb{R}_{+}, \lambda \in I$. 
THEOREM 7.2. We suppose that

(i) the conditions $\left(c_{1}\right)-\left(c_{6}\right)$ and the Theorem 3.1 are satisfied;

(ii) $K \in C\left(\mathbb{R}_{+}^{4} \times I \times \mathbb{R}, \mathbb{R}\right)$, has continuous derivative $\frac{\partial K}{\partial u}$ and there exists $q \in$ $(0,1)$ such that

$$
\int_{0}^{x} \int_{0}^{y}\left|\frac{\partial K}{\partial u}\right| e^{\tau(s+t)} d s d t \leqslant q e^{\tau(x+y)}, \forall x, y \in \mathbb{R}_{+} .
$$

Then the solution of the equation (7.1) is differentiable with respect to $\lambda$ and $\frac{\partial u}{\partial \lambda} \in C\left(\mathbb{R}_{+}^{2} \times I, \mathbb{R}\right)$.

Proof. In what follows we use the fiber contraction theorem (Theorem 7.1).

Let $X:=C\left(\mathbb{R}_{+}^{2} \times I, \mathbb{R}\right)$ and the operator $A: X \rightarrow X$ defined by

$$
\begin{aligned}
A(u)(x, y, \lambda):= & \text { the second part of }(6.1 \mathrm{i}) \\
A(u)(x, y, \lambda)= & g(x, y, h(u)(x, y), \lambda) \\
& +\int_{0}^{x} \int_{0}^{y} K(x, y, s, t, u(s, t, \lambda), \lambda) d s d t, \forall x, y \in \mathbb{R}_{+}, \lambda \in I .
\end{aligned}
$$

Let $u(x, y, \lambda)$ be the unique fixed point of $A$. We suppose that there exists $\frac{\partial u}{\partial \lambda}$. Then from (7.1) it follows that

$$
\begin{aligned}
\frac{\partial u(x, y, \lambda)}{\partial \lambda}= & \frac{\partial g(x, y, h(u)(x, y), \lambda)}{\partial \lambda} \\
& +\int_{0}^{x} \int_{0}^{y} \frac{\partial K(x, y, s, t, u(s, t, \lambda), \lambda)}{\partial u} \cdot \frac{\partial u(s, t, \lambda)}{\partial \lambda} d s d t \\
& +\int_{0}^{x} \int_{0}^{y} \frac{\partial K(x, y, s, t, u(s, t, \lambda), \lambda)}{\partial \lambda} d s d t
\end{aligned}
$$

In what follows we denote

$$
w(x, y, \lambda)=\frac{\partial u(x, y, \lambda)}{\partial \lambda}
$$

then we have

$$
\begin{aligned}
w(x, g, \lambda)= & \frac{\partial g(x, y, h(u)(x, y), \lambda)}{\partial \lambda} \\
& +\int_{0}^{x} \int_{0}^{y} \frac{\partial K(x, y, s, t, u(s, t, \lambda), \lambda)}{\partial u} w(s, t, \lambda) d s d t \\
& +\int_{0}^{x} \int_{0}^{y} \frac{\partial K(x, y, s, t, u(s, t, \lambda), \lambda)}{\partial \lambda} d s d t .
\end{aligned}
$$

This relation suggests to consider the following operator

$$
\mathscr{D}: C\left(\mathbb{R}_{+}^{2} \times I, \mathbb{R}\right) \times C\left(\mathbb{R}_{+}^{2} \times I, \mathbb{R}\right) \rightarrow C\left(\mathbb{R}_{+}^{2} \times I, \mathbb{R}\right)
$$


where

$$
\mathscr{D}(u, w)(x, y, \lambda):=\text { second part of (7.5) }
$$

for all $x, y \in \mathbb{R}_{+}$and $\lambda \in I$.

The operator $\mathscr{D}$ is a contraction with respect to $w$, indeed

$$
\begin{aligned}
& |\mathscr{D}(u, w)(x, y, \lambda)-\mathscr{D}(u, \bar{w})(x, y, \lambda)| \leqslant \int_{0}^{x} \int_{0}^{y} \mid \frac{\partial K(x, y, s, t, u(s, t, \lambda), \lambda)}{\partial u} w(s, t, \lambda) \\
& \quad-\frac{\partial K(x, y, s, t, u(s, t, \lambda), \lambda)}{\partial u} \bar{w}(s, t, \lambda) \mid d s d t \\
& \quad \leqslant \int_{0}^{x} \int_{0}^{y}\left|\frac{\partial K(x, y, s, t, u(s, t, \lambda), \lambda)}{\partial u}\right||w(s, t, \lambda)-\bar{w}(s, t, \lambda)| d s d t \\
& \quad \leqslant \int_{0}^{x} \int_{0}^{y}\left|\frac{\partial K(x, y, s, t, u(s, t, \lambda), \lambda)}{\partial u}\right||w(s, t, \lambda)-\bar{w}(s, t, \lambda)| e^{-\tau(s+t)} e^{\tau(s+t)} d s d t \\
& \leqslant\|w(s, t, \lambda)-\bar{w}(s, t, \lambda)\| q e^{\tau(x+y)} .
\end{aligned}
$$

Then we have

$$
\|\mathscr{D}(u, w)(x, y, \lambda)-\mathscr{D}(u, \bar{w})(x, y, \lambda)\| \leqslant q\|w(x, y, \lambda)-\bar{w}(x, y, \lambda)\|, \quad q \in(0,1)
$$

Then the operator $\mathscr{D}$ is a contraction and has a unique fixed point $w, \mathscr{D}(u, w)=w$. If we take the operator:

$$
\begin{gathered}
B: C\left(\mathbb{R}_{+}^{2} \times I, \mathbb{R}\right) \times C\left(\mathbb{R}_{+}^{2} \times I, \mathbb{R}\right) \rightarrow C\left(\mathbb{R}_{+}^{2} \times I, \mathbb{R}\right) \times C\left(\mathbb{R}_{+}^{2} \times I, \mathbb{R}\right) \\
B(u, w)=(A(u), \mathscr{D}(u, w)),
\end{gathered}
$$

from Theorem 7.1, we have that $B$ is a Picard operator. This implies that the sequences

$$
\begin{aligned}
& u_{n+1}:=A\left(u_{n}\right) \\
& w_{n+1}:=\mathscr{D}\left(u_{n}, w_{n}\right), n \in \mathbb{N},
\end{aligned}
$$

converge uniformly to $\left(u^{*}, w^{*}\right) \in F_{B}$, for all $u_{0}, w_{0} \in C\left(\mathbb{R}_{+}^{2} \times I, \mathbb{R}\right)$.

Let $u_{0}(x, y, \cdot) \in C^{1}(I)$ and $w_{0}:=\frac{\partial u_{0}}{\partial \lambda}$. Then by induction we prove that

$$
w_{n}=\frac{\partial u_{n}}{\partial \lambda}
$$

Therefore, $\left(u_{n}\right)$ converges uniformly to $u^{*}$ and $\left(\frac{\partial u_{n}}{\partial \lambda}\right)_{n \in \mathbb{N}}$ converges uniformly to $w^{*}$. From the above converges it follows that there exists $\frac{\partial u}{\partial \lambda}$ and $\frac{\partial u}{\partial \lambda}=w$. 


\section{REFERENCES}

[1] S. S. DRAGOMIR AND N. M. IONESCU, The Wendorff type lemma and applications (I), Itin. Seminar on functional equations, approx. and convexity, Cluj-Napoca, 1987, 135-142.

[2] S. S. Dragomir And N. M. IONESCU,, On a nonlinear integral inequalities in two independent variables, Studia Univ. Babeş-Bolyai, Mathematica, 34 (1989), 11-17.

[3] M. W. Hirsch And C. C. Pugh, Stable manifolds and hyperbolic sets, Proc. Symp. in Pure Math., AMS, 14 (1970), 133-143.

[4] V. Mureşan, Functional-Integral Equations, Mediamira, Cluj-Napoca, 2003.

[5] B. G. PAChPATte, On Volterra-Fredholm integral equation in two variables, Demonstratio Mathematica, 40, 4 (2007), 839-852.

[6] I. A. Rus, Picard operators and applications, SFPT, Babeş-Bolyai Univ., Cluj-Napoca, 1996.

[7] I. A. Rus, Generalized Contractions and Applications, Cluj University Press, Cluj-Napoca, 2001.

[8] I. A. Rus, Picard operators and applications, Scientia Mathematicae Japonicae, 58, 1 (2003), 191219.

(Received October 13, 2008)

Nicolaie Lungu

Technical University of Cluj-Napoca

Cluj-Napoca

Romania

e-mail: nlungu@math.utcluj.ro

Ioan A. Rus

Babeş-Bolyai University

Cluj-Napoca

Romania

e-mail: iarus@math.ubbcluj.ro 